Translating tweets in the soccer industry: Identity management and visibility in a global game

Roger Baines

University of East Anglia, UK 


\begin{abstract}
Migration has created exceptionally diverse communities within many professional soccer leagues. These diverse communities then interact with global audiences, yet the key challenge of communication across languages has not previously been the focus of academic attention. This study investigates Twitter translation practice within this highly commercial industry, drawing from questionnaire and interview data from the perspective of translation providers, figures in the soccer industry, and from migrant player and club tweets. The findings reveal tensions between global and local identities as soccer players, soccer clubs, and governing organizations manage identity performance and economic potential across language barriers on social media. These tensions foster debate about cross-language communication on social media in the soccer industry and extend existing work on social media and translation, in particular with regard to professional practice, fluency and the visibility of translation and translators in this unusual professional setting.
\end{abstract}

Keywords: Twitter, identity management, soccer, translation practice, fluency and visibility 
The intersection of professional translation in social media and the highly public relations (PR) conscious global industry/culture of soccer raises questions for both sport and communication studies and translation studies; questions about identity management and performance on social media, about tensions between global and local identity, and about fluency and the visibility of the translator and translation.

In the soccer industry, exceptionally diverse professional communities interact with global audiences, yet the challenge of communication across languages has received very little academic attention. Sport and communication scholarship has not hitherto turned the spotlight on this critical area for migrant athletes and coaches, their clubs, associations and fan-bases. Nor has research in sport and migration, or sport and globalization developed in this area. A parallel gap in scholarship exists in relation to social media in translation studies as the sole major study of translation and online social media appeared only in 2017.

While the migration of athletes has soared in many professional sports since the 1990s (Maguire \& Falcous, 2010), the increasing migration of soccer players and coaches (Poli, 2014) creates distinctive and diverse multilingual professional communities. This study focuses on soccer because of this distinctive diversity, and also because it is a hugely popular professional sport on a global scale (for example, the 2014 FIFA World Cup competition reached 3.2 billion viewers [Associated Press, 2015]). Tweets translated within the soccer industry thus attract more fan and media interest than in any other sport. Although increased migration affects both elite and non-elite athletes (Carter, 2011), the focus here is on elite soccer contexts because it is only at this level that investment in professional translators tends to occur.

Automatic (machine) translation is commonly used, in particular by fans, to translate tweets, notwithstanding the relatively limited quality that can be achieved. However, this investigation considers translation by professional human translators rather than automatic 
translation, to address the gap in research into professional social media translation practice. Focusing on professional human translation also uncovers the challenges for translators working in the soccer industry/culture, and reveals how professional translators navigate their way through issues related to identity management, to global and local identity, and to fluency and visibility on Twitter.

\section{Research Context}

\section{Twitter and Sport}

There is now considerable scholarship in English devoted to Twitter in the sport context. Topics range from assessing the potential of Twitter for fan/club-athlete interaction (e.g., Pegoraro, 2010), to differences between Twitter and traditional sports journalism (e.g., Price, Farrington, \& Hall, 2013) through analysis of how sports stars can use Twitter as a promotional tool (e.g., Hambrick, Simmons, Greenhalgh, \& Greenwell, 2010; Pegoraro \& Jinnah, 2012) and to build athlete identity (e.g., Sanderson, 2013). In a Communication \& Sport Twitter research forum essay Hutchins (2014) acknowledges the pivotal role of social networking platforms in contemporary sport. He also observes repetitiveness in some research into social media and sport, noting both the popularity of sport on Twitter and straightforward data collection as causes. He calls for closer examination of Twitter's commercial status and a more central focus in future research on commodification.

\section{Twitter and Translation}

Tweets composed in a wide range of languages and tweets crossing language barriers create the conditions for tweet translation. Even if English is the global lingua franca, most of the world's population does not have first or second language access to English (Eriksen, 2014), and most of the Twitter-using population does not compose tweets in English (Bennett, 2011; Mocanu, Baronchelli, Perra, Gonçalves, Zhang, \& Vespignani, 2013). High levels of heterogeneity in the nationalities of athletes, coaches and fans, combined with high- 
profile media coverage, give professional sports contexts a particularly rich character in tweets that interest audiences in multiple languages. Migrant athletes and coaches, for example, regularly work in contexts where they are not proficient in the host country language(s). They thus publish tweets in their first language:

\{Insert Figure 1 here with caption $\}$

Or produce non-fluent tweets in the host language:

\{Insert Figure 2 here $\}$

Or tweet in both their first language and the host language to communicate with monolingual and multilingual fans:

\{Insert Figure 3 here $\}$

Multilingual tweeting by athletes and coaches, and monolingual tweeting in a language which is not the principal language of the host country, are supplemented by multilingual tweets from sport clubs, media outlets, and transnational governing bodies.

Despite the global popularity of Twitter, the generation of tweets in multiple languages, and the demand for translation, there had been minimal research explicitly focused on online social media and translation (e.g., Desjardins, 2013; Kelly, 2009) until Desjardins' Translation and Social Media (2017). This reflects the general lack of visibility and awareness of Twitter translation. This can be observed in non-sports news media, for example. The translation of tweets is part of the process of mediating news events across 
language barriers that takes place all the time in international news, yet tweet translation is just as invisible to the news consumer as, for example, the translation of interviews, eyewitness accounts, or news agency reports. This is in keeping with prevailing attitudes to translation and interpreting outside the discipline of translation studies and the language professions. Major works and handbooks on social media (e.g., see Billings \& Hardin, 2014; Fuchs, 2017; Georgakopoulou \& Spilioti, 2015) have entirely overlooked translation despite their international, globalized scope. The present investigation is thus innovative both in the sports communication field and in the translation studies field.

\section{Twitter and Branding}

Considerable research demonstrates the value of sports stars using social media to promote their brand (see Korzynski \& Paniagua, 2015; Parganas, Anagnostopoulos, \& Chadwick, 2015) but there are high risks. Social media posts can present a threat to the strength of soccer player or club brands as there is a well-developed sensitivity to any media coverage that could damage positive PR narratives, and consequently affect reputation and economic strength. This is reflected in the ways in which soccer clubs and professional organizations educate players about social media use. See, for example: the standard section in players' codes of conduct relating to social media use, forbidding comments that can be construed as negative (Johnson, 2016); social media guidance provided by the players' union in the UK, the Professional Footballers Association (PFA); and club and governing body fines for inappropriate tweets. Other kinds of corporations exert similar power over social media content; for example, see Vaast and Kaganer (2013), and ACAS (2018). This high sensitivity to the dangers of brand damage is critical to tweet translators' work in the industry. 


\section{Translation, Brands, and Glocalization}

This investigation underlines the fundamental relationship between brand and the translation of tweets in the market-led soccer industry/culture in line with Hutchins' comment that research into Twitter in sports contexts needed to focus more on commodification. For Gilchrist: "The modern sport star (...) is (...) both cultural product (as a brand to be sold) and process (part of the chain of advertising and brand or product endorsement that underpins the regime of capital accumulation ..." (2005, p. 126). Soccer stars, clubs, and governing bodies are commodities which have a brand that needs to be protected. In translation, PR affects the very first decision about the method for translating tweets: human or automatic online translation. Twitter users can click to access an automatic translation provided by Microsoft's Bing Translator in over 40 languages. This is how most tweets are translated by individual users. However, players, player representatives, clubs and governing bodies usually exercise more care. If a particular tweet is needed in another language they tend to use professional human translators, unless the tweeter is sufficiently fluent to translate/compose a similar tweet (as in Figure 3). This is because PR-conscious professionals wish to avoid the potential brand damage of a poor quality automatically translated tweet.

Commercial objectives reflect the soccer industry's dominant ethos and permeate translation practice. Although the value of professional translation is by no means generally accepted in the soccer industry, it is nonetheless an industry where, in most contexts, resources could be found to fund translation. It is notable that the majority of the minimal translation studies work on social media and translation relates to translation in nonprofessional contexts, in contexts where funding for translation is not necessarily available (fan translation, crisis management, activism etc.) Desjardins (2017) reviews existing work, arguing that online social media translation does not always coincide with translation for a commercial purpose, as in fan translation, activism etc. The translation of tweets in the soccer 
industry, however, does entirely coincide with translation for a commercial purpose. This commercial underpinning of translation activity, and the destabilising effect it has on the control of media managers over clubs' self-confirming narratives, can also be seen in the translation of press interviews and press conferences in the soccer industry (see Baines, 2018). The management of the brand identity of players, and of clubs, as communicated through Twitter, can also be compromised by translation, and by expression in a foreign language. When thinking about the interplay between the global marketing context of the soccer industry, and local identity as expressed in the use of language, and the translation of that language, glocalization is an important concept.

Debating the way in which globalization became misunderstood as a homogenizing process of cultural imperialism rather than simultaneous and inter-penetrative local and global processes, Robertson (2012) puts forward glocalization as a refinement of globalization. For him, glocalization better captures the interweaving of the local and the global:

The idea of glocalization in its business sense is closely related to what in some contexts is called, in more straightforwardly economic terms, "micro-marketing": the tailoring and advertising of goods and services on a global or near-global basis to increasingly differentiated local and particular markets (p. 194).

This is a sound basis for examining the ways in which glocalization to differentiated markets can be detected in how those managing Twitter accounts deal with translation and non-fluent expression in the host language. Applying the concept of glocalization to sport and the successful reach of major brands in global markets, Giulianotti and Robertson (2007) argue that rather than seeking to neuter cultural difference through a strategic global 
uniformity, the transnational corporations that major sports teams/franchises now are "have acknowledged that securing a profitable global presence necessitates operating in the languages of the local" (p. 34). Language is a major characteristic that can mark out or express the local in a particular context, define a context as local or, potentially, in the use of the common global lingua franca of English, be symptomatic of processes which impose cultural homogeneity and sameness. Glocalizing strategies, of course, play out in different ways for different businesses, for different brands, but the tension and interplay between the local and the global resonates strongly in the framework of global sport brand identity when looking at tweets by migrant soccer players and coaches and their translations/tweets expressed with a lack of fluency in the host language.

\section{Identity Performance and Management}

Identity and identity performance are crucial to high-profile sports stars and organizations in creating and maintaining brands. Studies of celebrity tweeting (e.g., Marwick \& boyd, 2011) and sports celebrity tweeting (e.g., Lebel \& Danylchuk, 2012) give accounts of the identity management that soccer clubs, players, agents, and governing bodies want to practice but which can be jeopardized in translation, or in the composition of tweets in a language in which the player or coach is not proficient. Goffman's seminal The Presentation of Self in Everyday Life (1959) argues that the performance of the self is a continuous presentation before a certain group of people designed to have an influence on those observers. In contrast to this societal front stage "performance" is the area of the backstage where the individual is no longer pressurized to maintain a public performance (Leary, 1995).

Goffman's self presentation theory has been used to explain the appeal of social media for celebrities, including sports stars, because social media provides direct access to the backstage dimensions of their lives without any mediation by traditional media (Hutchins, 
2010). In most cases this "backstage" access afforded by Twitter is an illusion because what is performed is carefully managed identity for self-promotion. Kytölä and Westinen (2015), writing about Finnish soccer player Mikkel Forssell switching on Twitter into a language (or variety) generally not thought to belong to him, argue that there is a re-negotiation by the audience of "the relation between speaker, place and language in seeking to determine who has the right to use which linguistic resources to/with whom and where" and note that “authenticity and normativity (correctness, appropriateness and expertise) become key issues in these negotiations" (p. 17). Objections by those reading and commenting on Forssell's use of "gangsta" language on Twitter centered on the inauthenticity of that language in relation to the norms of Forssell's habitual communication to which readers will have been exposed through other media, including spoken media. Thomas (2014) notes that the intimacy and immediacy of Twitter's interface "increases the potential for a star's tweets to be interpreted as an indicator of an authentically ordinary star voice" (p. 253) but a common assumption is that most celebrities do not compose their own tweets. For example, Desjardins (2017) notes that in celebrity contexts disclosure as to who is the actual author of an online social media account is rarely given. Migrant players or coaches, and their agents, may be invested in appearing authentic, appearing to be themselves, through their language use on Twitter to cultivate a strong relationship with fans. This objective will, in most cases, have a commercial dimension (Pegoraro \& Jinnah, 2012).

\section{Translation and Visibility}

Examining the translation of tweets in the soccer industry through a translation studies lens reveals another common point of contact with identity performance and management which is key to professional translation practice: visibility. As noted above, what is visible backstage on Twitter is often stage-managed by high-profile soccer players 
and their agents, as it is by other celebrities. Translation usually becomes visible when a lack of fluency in the target language (the language being translated into) highlights the fact that a text has been translated (in the same way that a lack of fluency in the host language foregrounds "foreignness"). Fluency in the target language is usually a professional objective for a professional translator, a norm which renders the translator and the translation process invisible. Achieving fluency in translation is a strategy which reflects the soccer industry's commercial objectives. Translations which provide a smooth reading experience are better for reputation and economic standing than translations which are difficult to process or which contain errors or inaccuracies. This is the same commercial imperative that is applied to the localization of digital products and digital platforms for consumption in diverse linguistic and cultural contexts (Schäler, 2010). This commercial imperative is also found in the AngloAmerican literary publishing industry where translations into English which read fluently and are adapted to the receiving culture's norms are favored because they sell better (Venuti, 2008).

The fluency norms of professional translation practice described here have, however, been contested on ethical grounds in translation studies, in particular by Venuti (2008). A translation strategy which prioritizes fluency in the target context over fidelity to the source text is called a domesticating/acculturating strategy. Venuti, among others, argued that when such a strategy is used the source culture and the translation profession are denied visibility and recognition (e.g., see Bassnett, 2005; Desjardins, 2017; Venuti, 2010). Such academic concerns are, however, rarely observed in professional practice where fluency has high value, and recognition of the worth of translators and translation generally has low value. A lack of fluency in poor quality translated texts can adversely affect both the reputation and economic standing of the client who commissioned the translation, and the reputation and job security of the translation provider/translator (see Desjardins, 2017; Drugan, 2013). Consequently, 
fluency in the translation of tweets in the soccer industry should be the norm. However, in relation to identity performance in the backstage of Twitter misspellings and grammatical errors, which are not usually indicators of fluency, are commonly recognized as a sign of authenticity (Marwick \& boyd, 2011). This tension between the value of fluency and the value of authenticity, both of which contribute to the development of the economic value of a player or coach's brand, link up with questions raised above in the context of glocalization, and reflect decisions that translators of tweets and their clients in the soccer industry have to take.

Based on the studies, theory, and research detailed above, the following three research questions guided the current investigation:

Research Question 1 (RQ1): In the framework of Robertson (2012), and Giulianotti and Robertson (2007) on glocalization, what does the picture built up by interviewees and questionnaire respondents reveal about translation, identity and processes of glocalization in the soccer industry?

Research Question 2 (RQ2): Following discussion of work by Goffman (1959), Gilchrist (2005), Hutchins (2014), and Kyötöla and Westinen (2015), what do interview and questionnaire responses reveal about the pressures of brand identity management on translation practices on Twitter in the soccer industry?

Research Question 3 (RQ3): Considering the work of Venuti (2008), what do interview and questionnaire responses reveal about fluency and the visibility of the translator/translation process in relation to the translation of tweets in the soccer in industry?

\section{Method}

Once initial interviews had been conducted, it was possible to focus in on particular issues in questionnaires relating to identity management on Twitter in translation and visibility in translation. The method of enquiry was interpretive and 
qualitative. The data presented here consist of interview and questionnaire responses, and some tweets. The interviews are part of a larger holistic investigation of policy and practice relating to communication across language barriers in the soccer industry in Europe for which three soccer agents, two soccer club media managers, four former migrant soccer players, eight soccer club player liaison/welfare officers, and 10 translators/interpreters have so far been interviewed.

However, the data interpreted below come from comments made about social media translation in a subset of the interviews described above: principally five interviews with professional translators, but also two with former migrant players (one French, one Spanish) who worked in the English Premier League (EPL), one with an EPL club media manager, and one with a soccer players' agent. The translators represented a diverse range of languages and working contexts: of the five translators interviewed, one translated into French from English; the other four translated into English from either French and Italian, French and Spanish, or Spanish and Portuguese. They work for players, coaches, and clubs in the highest leagues: the English Premier League, "Ligue Un" in France, "Serie A" in Italy, and "La Liga" in Spain.

Five of the interviews were conducted in person, four via Skype. Interviews were used because the investigation was concerned with facts, descriptions and opinions recalled by interviewees about translation in the soccer industry. Semi-structured interviews were used because they provided the flexibility to adapt questions to suit the interviewee and to pursue other questions that emerged in the interaction. The open interview questions about translation and social media were designed to extract descriptions of experience and to home in on issues and challenges the translators had experienced in the translation of tweets in the soccer industry.

Further data were collected via five questionnaires focused specifically on the 
translation of tweets submitted to five translators. These were exchanged via email with four of the professional translators interviewed plus one other who worked for a governing body who became available after the interviews. The questionnaires used open-ended questions building on the material collected in the interviews which had raised questions about: identity management and the illusion of backstage performance on Twitter through translation; and fluency and translation/translator visibility.

The nine interviews were transcribed for analysis. The data in the interview transcription and the questionnaires were then grouped by theme and relevant passages extracted for interpretation under the headings of the research questions. Both the interview and the questionnaire were piloted with the first respondent in each category and their design informed by Hale and Napier (2013). A final data set consisted of tweets collected from the public domain. In addition, tweets provided by research respondents were interpreted, as were tweets by migrant players and by clubs which were used to illustrate specific points that emerged from the analysis of the nine interviews and five questionnaires.

Goffman's work on identity and identity management as related to performance and Venuti's work on the ethics of the translator's invisibility informed the collection of questionnaire data and were used for thematic qualitative analysis of all the data. However the thread of enquiry around glocalization did not feature explicitly or implicitly in the interviews and questionnaires, it only emerged once the data was being analyzed which is when Robertson (2012), and Giulianotti and Robertson's (2007) work was investigated. Gaining research access to members of the soccer industry, especially at the more elite levels, is particularly difficult because of the industry's heightened wariness around any potential damage to brand and reputation (Roderick, 2006). Consequently, identifying and agreeing interviews with some of the former players, agents, and media managers who provided some of the data relied on a series of contacts provided by interviewees and a 
snowballing method. The translators were less difficult to identify and reach but a similar snowballing method was used to gain access. All questionnaire respondents and interviewees gave permission for the data to be used anonymously. In consultation with the translators who have signed professional non-disclosure agreements with their clients, some examples have been adjusted to further protect anonymity, thus underlining the commercially sensitive character of the contexts in which these translators work.

\section{Findings and Discussion}

The high sensitivity to the dangers of brand damage, and an awareness of the increased risks to reputation when using a foreign language or using translation, are confirmed by the following comments from former migrant players, an English Premier League club media manager, and a soccer players' agent/representative:

Your credibility could suddenly just go, your words could be taken out of context and that could go viral (Former player 1, 2017);

There are examples of people, there are so many, players who go just to Google translator [sic] and they will just get the translation and paste it in Twitter, "don't do that, that's completely the opposite meaning of what you wrote!" (Former player 2, 2017).

We have to have complete trust in the people translating ... We have people on contracts translating basically all our content into our Twitter feeds across the world but if I'm looking at our Weibo feed or our Twitter feed into Mandarin, I obviously haven't got a clue (Media manager, 2015). 
We would encourage a foreign player in the early stages to send any draft tweets to us because I am very aware that one erroneous tweet can ruin a career ... Commercially the player needs to develop an English following as soon as they can if they want to maximize their career earning opportunities as much as possible so it's a balance between starting as soon as possible but not so soon that it's disastrous (Soccer agent, 2015).

The translators' perspective reinforces this state of caution and awareness of risk to reputation, characteristics which are heightened in translation:

The last thing that you want is for the club or player to receive abuse because of a typo or poor translation. Once a tweet appears, it can be nigh impossible to delete it entirely as people are so ready to screenshot and grab anything that they think might be useful for trolling, ridiculing or anything else (Translator 4, 2017).

The account of the research context for this investigation into the translation of tweets in the soccer industry demonstrates how interconnected the issues are. There is consequently necessary dialogue between all the research questions. Glocalization provides a thread through much of the discussion signaling its importance to this investigation. The first research question functions as an umbrella for all the questions this investigation poses.

Regarding the first research question (RQ1), the architecture of different types of soccer club Twitter account have an effect on how the glocalizing process works. There are typically two types: bilingual accounts where tweets are published together in two languages on a single bilingual feed (Figure 4), and monolingual accounts where tweets are published 
separately in parallel accounts and the tweet translations are presented independently of the source text (Figure 5).

\section{\{Insert Figure 4 here $\}$}

\{Insert Figure 5 here, 2 images: 5 i followed by 5 ii each with a caption $\}$

There is also a third type where there is no attempt to reach a more global market and tweets are only published in the host language. Both types of account illustrated above demonstrate a club's desire to move beyond the local context and communicate its brand to a wider more global audience through translation. There is however a difference in the ways in which the two types of account exhibit the "simultaneity and inter-penetration" of glocalization that Robertson (2012, p. 194) describes. The single bilingual account in Figure 4 does this most clearly. Fiorentina retains its identity as an Italian Serie A club but shares that identity with a wider English-speaking audience in translation. It visibly takes its local property of Italian language into a more global context. In contrast, Marseille communicates independently with separate parallel audiences in their parallel French and English accounts. Marseille's local identity is not compromised and the effect of reaching a wider audience through the use of English is doubtless achieved. There is more scope in the latter process for the translation to differ, for content to be developed independently. In the case of the parallel account, an English-language version of Marseille's Twitter feed is developed. Content is more or less simultaneous. It is not visibly inter-penetrated in the way content is in the single bilingual account but it remains an example of the heterogeneity of glocalization. Transnational soccer organizations take a similar glocalizing approach to that seen in 
Marseille's Twitter accounts. UEFA, for example, the European Soccer governing body, provide separate parallel monolingual content which can be quite independent:

We are given the freedom to create our own content in French, content that will interest French people and French-speaking people, content that fits our audience. Sometimes, we base our tweets on English tweets to get an idea. It's not "translating" per se, but it's as close at it gets (Translator 5, 2017).

So, UEFA, and many clubs with global ambitions use parallel Twitter accounts to glocalize by shaping "local" content for specific language audiences worldwide. The translators in this study provided a number of comments/examples of tweets which were composed in English based on a tweet in another language rather than translated by closely following the source language lexis and grammar.

It's such a small piece of text, often only a few words, that it's hard to get context and it might be easier to get that message across in $X$ language in five words, you might have to write something completely different to get the same meaning across (Translator 2, 2015).

Following a high scoring defeat in which a Spanish club conceded six goals before then scoring four, the club tweeted the headline of a match report: "Si compite es muy bueno, pero si no..." (literal translation: "If it competes it is very well but if not..."). The translator reported considering "When the team competes it does very well, but when it is not at it, it doesn't..." as a translation but assessed it as too long for Twitter. Instead the sense, and the 
brevity and style of the original, were captured in a new tweet "Spanish Club in Jekyll and Hyde performance" (Translator 3, 2017).

Lexical choices frequently carry properties that link them to specific cultural contexts. Those lexical choices can therefore mark out a local context. Categorizations of register can be a way of differentiating one stretch of language from another in terms of variation in context, relating to the language user and/or language use. The translators noted a specific area of contextual variation that derives from the norms of language use in relation to technical language. Describing injuries in technical detail is not the norm in English (e.g., see Jones \& Steckelmacher, 2012) and technical language is regularly subject to either omission or simplification in translation into English. In response to a question about register shifts, these two comments were collected about club Twitter accounts:

Player injuries is one of the prime examples of this. My thinking is: the tweet is being put out there to inform fans of an injury, therefore it's better to render the tweet in language that the general public are going to understand. I've tried translating literally - and sometimes it's necessary for official statements - but it really is difficult (Translator 2, 2017).

I prefer to talk about calf, thigh strain etc. but some clients want the medical term even in English. Personally, it jars and I challenge any non-medical professional to decipher it (Translator 1, 2017).

Thus translators report adapting the norms of local language use in the source language to the norms of local language use in the target language of English. Avoiding the production of texts which would read awkwardly in English enables the communication to be smooth and 
fluent and thus does not risk negative PR. By reflecting local norms, the translators are glocalizing by differentiating the various clubs' Twitter communication in a way that suits the local context.

In contrast, the translators' approach to hashtags in Twitter accounts does not represent a glocalizing approach. The question of effective indexing for soccer tweet translators working with hashtags underscores how the non-linear production and reading of tweets and posts on social media in general differs from most other kinds of texts. This property means that hashtags, which are generally designed in the commercial context of professional soccer to reach as many consumers as possible, are frequently left untranslated because a translated hashtag would most likely not achieve comparable recognition and reach to the original hashtag (Desjardins, 2017) reducing the size of its comparable audience. This is a significant problem for the kind of sponsored or corporate content found in the soccer industry. Unlike hypertext links, hashtags are not necessarily a route to related content because a hashtag is often quite broad in meaning and connotation. It can therefore group together very disparate content from a wide range of sources. One translator commented:

The clubs will try to use a hashtag that works in all languages. For example, Inter put \#FCIM at the end of all their tweets (the initials of the club). It doesn't need translating but serves to collate all Inter-related tweets in one place. If they tweet about a world event, such as Holocaust Day, they'll use the Italian hashtag and we'll use the English one. But I would never translate \#ForzaJuve to \#ComeonJuve, for example (Translator 1, 2017).

\#ComeonJuve would not have the recognition and reach of \#ForzaJuve and so would not achieve similar indexing and topic popularity, and it is worth noting that amongst soccer fans 
the use of "Forza" outside Italy is to some extent already globalized. It is familiar to many and, for some, has become part of their vernacular as a performative example of identity as a knowledgeable fan following Italian soccer. This property gives the Italian hashtag yet more global reach. In contrast, a similar but more locally coded hashtag in the following tweet (Figure 6) by West Ham's Manuel Lanzini would not have the same global reach as \#ForzaJuve:

\{Insert Figure 6 here $\}$

"COYI" stands for "Come on You Irons" which is a standard chant by West Ham fans. "Irons" is shorthand for "hammers" and both are nicknames for the club. "COYI" could not be adapted in the way that the technical language described above could be because meaning and associations are too densely packed for a suitable translation to be found. A further example was provided in this account by one of the translators:

I was tweeting live during the game and as Seville started their comeback from 4-1 down, the following hashtag started to appear at the end of the match tweets: \#DicenQueNuncaSeRinde (They say that you should never give up) (Translator 3, 2017).

The translator considered translating this club motto as “\#it's not over til it's over" but decided not to for the reasons of limited reach discussed above. The translator acknowledged that there had to be a certain cultural loss because the English language audience "did not get the feeling that this dramatic comeback was linked to one of the club's values" (Translator 3, 2017). This is thus another example of a local element that 
could not be carried across to a differentiated context. A final illustration from UEFA explained by one of the translators shows an English language hashtag proving to be dominant in the marketplace:

Our main UEFA competition is the UEFA Champions League that has a hashtag all English-speaking fans recognize: \#UCL. It has really become a brand in English. In French, we have been using \#LdC consistently for years for "Ligue des champions". A few other accounts use it but it hasn't really caught on. So we've started adding \#UCL on top of \#LdC to be able to have more visibility (Translator 5, 2017).

"More visibility" is synonymous with more commercial reach. The apparent policy within the soccer industry of creating hashtags which are universally understood, or of avoiding the translation of hashtags completely, would appear to reflect a less glocalizing strategy than that explored in the translation of technical language relating to injuries. In fact, the strategies could be read as more globalizing where globalizing is understood as a homogenizing process of cultural imperialism. The exploration of glocalizing processes in professional tweet translation in the soccer industry reveals that such processes can be detected even if they manifest themselves in different ways in different contexts. However, it appears that the nonlinear property of hashtags leads to commercial decisions to employ a more globalizing/homogenizing approach than the glocalizing approach used for text content. Commercial considerations are similarly prominent when looking at translation and the management of backstage identity on Twitter.

Regarding the second research question (RQ2), as discussed above, the investment of migrant players or coaches, and their representatives, in giving the impression of being authentic through their language use on Twitter has the objective of cultivating a strong 
commercial relationship with fans. Translation adds an additional layer to the performance of backstage identity. All migrant soccer players or coaches who tweet in a language which is not their first language, or have their tweets translated, place themselves in a position where their use of a language that is foreign to them naturally becomes a prominent feature of their communication, and an aspect of identity that can be staged. The following two contrasting examples both demonstrate a desire to use translation to manage backstage image. The first comes from a translator who was supporting a group of migrant players at an English Premier League club:

I was called upon to translate tweets by one player at various hours of the morning, tweets that were in Spanish that he wanted idiomatically put into English. He was saying something in Spanish that had a certain nuance to it, he wanted to have a translation that had the same punch in English (Translator 6, 2015).

The translation objective in this case is quite clear and in line with professional expectations of fluency from a professional translator. Fluency in the foreign language projects a positive backstage identity which is beneficial in terms of PR and its associated commercial objectives.

The second case, however, is quite different. Here, the translator was explicitly asked to avoid the fluency sought in the first example:

The tweets that I translate have supposedly been composed by the football players/coaches that I'm working for. Whilst my other clients expect readable and natural English translations in tweets, in this instance, I'm asked to pitch the English used in my translations at the level of a foreign football manager, whose grasp of the 
English language may not be all that great. At times, I have been asked to avoid using a colloquial turn of phrase in a tweet purely on the basis that it would be beyond the grasp of the account owner. I recall suggesting the use of "Lady luck shone on...", which was rejected by the client (Translator 3, 2017).

An awareness that fluent English would pose a challenge to the audience in terms of their expectation of authenticity and normativity of expression can be observed in the instructions to the translator. The client's objective was to communicate what the fans might interpret as features of authenticity: "they want to try and avoid Twitter users commenting on tweets and saying things such as, "This was obviously not written by X"' (Translator 3, 2017). This concern for preserving authenticity and normativity of expression dictates a nonfluent translation. However much the backstage performance is managed on Twitter, many readers will have a sense of how well that player or coach can actually communicate in that host language through exposure to the player speaking, front stage, in a press conference or during a post-match interview where language performance cannot be adjusted in the way it can on social media. From this, readers will know how fluent that player or coach is in the host language. This awareness will play a part in the "negotiations" about authenticity and normativity that Kytölä and Westinen (2015) describe in their analysis of Mikkel Forssell's use of gangsta language in his tweets. Assessing authenticity in any non-fluent tweets which derive from press conferences is relatively straightforward. Any manipulation of such tweets by the translator/proofreader would be detectable because press conferences are now usually filmed and made available online, thus the original speech could be checked. What is more, press conference texts are texts spoken by the players/coaches. There is no gap between the front stage player/coach identity (idiolect) visible in the press conference and what is communicated backstage via Twitter. The intention to create an illusion is, however, evident 
in the translation of tweets and the proofreading of non-fluent tweets written in the host language where client instruction to the translator/proofreader has the objective of preserving the "authenticity" of expression in English, or preserving the tweeter's idiolect. Yet, generally, there is a clear suspension of disbelief because the backstage identity is, in most cases, constructed as the tweets have in all probability not been composed by the players/coaches (see Ruthven, 2016). This example of a desire for authenticity in translated tweets is expressed in a context, celebrity tweeting, within which there is a widespread belief that the authenticity of tweets is compromised. As this particular translator went on to add:

I believe that the feeling that the client has is that Twitter users want to see material that is written by the coaches themselves even though, in reality, everyone recognizes that the large majority of footballing personalities often don't think up/post their own tweets (Translator 3, 2017).

This is akin to practices in the entertainment industry, as described by, for example, Kaufman (2013) and Desjardins (2017). The desire to preserve the authenticity of constructed back stage identity is further unveiled through tweets composed in English where the translator is asked to review/proof-read rather than translate:

I have received tweets for editing and have been asked by my client not to correct certain turns of phrases which have been used by coaches/players in a press conference. I have also been asked for other tweets written in English as a foreign language not to upgrade the level of English too much, so that it is in keeping with the sort of English that the supposed author could feasibly produce. An example of this 
might be: "Today we made a good game" versus "Today we put in a good performance" (Translator 3, 2017).

Given that misspellings and grammatical errors are commonly recognized as a sign of authenticity on social media (Marwick \& boyd, 2011), then preserving a lack of fluency in translated or proofread tweets is in line with the commercial benefits that authenticity brings. This is not incompatible with the first example where it was argued that a fluent translation ("with the same punch") was good for PR. However, to turn back briefly to RQ1, the two examples described above - providing a fluent translation, and preserving a lack of fluency in translated or proofread tweets - both provide illustrations of different strategies of glocalization. The fluent example enables the migrant player to communicate with differentiated markets while the non-fluent example enables the migrant coach's local identity to remain visible as well as authentic. This brings the investigation to the question of visibility in translation.

Exploring the third research question (RQ3), the unusual example above of a lack of fluency actively sought by clients in order to sustain the illusion of authenticity in celebrity tweets is especially interesting in relation to visibility in translation studies. In terms of translation strategies, it is a rare example of foreignization. This is a strategy promoted by Venuti (2008) because it makes the translation process, the translator and the source language/culture, visible in a way that is highly unusual in most professional translation contexts and, in particular in commercial contexts. Yet, despite the strategy's rarity as a practice, it is motivated by precisely the same concerns with positive brand management as the more usual practice of fluent translation.

Translation/translator invisibility can be compounded by the rapidity of communication enabled by technology. Once a tweet is published immediacy is key to effective 
communication: "if you're working for a major client, and football clubs generally are, you better translate it quickly for the official account otherwise someone else will report it before you" (Translator 1, 2017). It is the tweets that need reacting to and so need translating fast which are most challenging for the professional translator in soccer. There are two principal contexts for live tweets: live press conferences where a manager or a player speaks to the assembled press, and live matches which will be the focus here: "There was certainly pressure to translate them quickly during matches as, if there's been a goal, the fans need to know fast!” (Translator 3, 2017). The speed with which tweets can be posted creates an expectation of high speed translation, in particular for users used to automatic online translation tools. This gives the impression that the translation has indeed been carried out simultaneously and automatically even if professional human translators are involved. By posting tweet translations rapidly translators thus reinforce their own invisibility (Desjardins, 2017). The pressure to translate quickly, however, has the potential to affect translation quality: "In some instances, this has seen me be less adventurous with translations, given that there is simply not enough time to check things before submitting” (Translator 3, 2017); "Increased chances of typos/meaning errors" (Translator 1, 2017); "a typo can reflect very poorly on a club's image which is not great" (Translator 2, 2017). For live matches, the challenge of creating quality fast translations of live tweets can be compounded by not being able to see what is being described:

As soon as they tweet in $X$ language, we need to go in English. This can be tricky as you want to be watching the game to know what's happened before you tweet, as going on their tweet alone would open up a lot of scope for error (Translator 2, 2015).

A series of technical challenges can mitigate against fast translation: 
Often you have to do 3 matches at a time (...) so it can often happen that X club, Y club and $Z$ club are playing at the same time. (...) You can't really get much context from a tweet so I'm guessing that what we actually put in English is often not the best description of what happened. (Translator 2, 2015).

Take scoring a goal, for example - there are so many ways to put the ball in the back of the net: you could thump it, dink it, slot it, smash it, fire it, thrash it, thunder it, sweep it, roll it home. You can't really pick one of those in full confidence without first having seen the goal, so that can be an issue if you're trying to follow a game and keep an eye on the Twitter feed at the same time (Translator 2, 2017).

This description is similar to the experience of video game translators who, more often than not, cannot see the game for the context they need to translate well (Mangiron \& O'Hagan, 2006). Errors created by a combination of time pressure or a lack of the right tools make the translation process, and by extension the translator, visible as well as affecting the reputation of the client and the translator/translation provider.

Visual literacy-based text on Twitter, in contrast, raises little debate about translator visibility because translation is largely not required. Images, video clips, and emojis represent social media content that is becoming increasingly important and requires users to be more and more visually literate (Desjardins, 2017). Emojis have now become a global phenomenon: in 2015, according to Evans (2015), over 6 billion emojis were being sent every day by over 90 percent of the world's online population, a global reach that surpasses that of any language (also Alshenqeeti, 2016). Emojis represent a global communication practice which transcends linguistic and cultural boundaries. The use of emojis in tweets in the soccer 
industry, as in other contexts, fulfils a globalizing function similar to the non-translation of English language hashtags without the linguistic imperialism. However, ascribing universality to emojis is not always a safe assumption. Danesi (2017), for example, devotes a chapter to cross-cultural variation in emojis explaining how different emojis may be subject to different historical and cultural associations of meaning. The use of emojis nonetheless remains at the globalizing (homogenizing) end of the globalizing/glocalizing spectrum, though the context in which they are used can affect their position on that spectrum. Here are two examples of emojis used by football clubs: one a Juventus pre-season tour tweet on their Italian Twitter account (Figure 7), the other a Bayer Leverkusen in-game tweet on their English language Twitter account (Figure 8):

\{Insert Figure 7 here with caption (translation)

\{Insert Figure 8 here $\}$

In each case, the emojis convey additional meaning that is not explicitly expressed in the tweet itself. Although the use of the "strong" emoji in the Juventus tweet is likely to be universally understood, it is employed within the club's Italian language twitter account which glocalizes the tweet it appears in towards an Italian-speaking audience. The use of the fire emoji in the Bayer Leverkusen example requires knowledge of the English language to decode the implicit reference to the expression "to be on fire" i.e. to be performing extremely well, though similar expressions may exist in other languages. The extra knowledge required dilutes the emoji's universality rendering its use less globalizing. Yet the emoji is used in the context of Bayer Leverkusen's English language Twitter account so the communication is already glocalized towards an English-speaking audience. Another interesting example is the 
use of national flag emojis. These are potentially universally understood but the "local" that is associated with certain flags can be problematic, as reported by the UEFA translator:

Emojis of country flags are really a must for us. This poses a few "political" problems sometimes because on Twitter, there's no flag for Scotland (just a Union Jack) or for Catalonia. When we tweet about Barça, we avoid using the Spanish flag emoji because we know it doesn't represent FC Barcelona. But we use it a lot for Real Madrid (Translator 5, 2017).

So, while emojis do not need translating and are generally universally understood, the context in which they are used, and/or the need for additional linguistic knowledge to decode the emoji can lend them a more glocalized character than might initially be assumed.

The fact that emojis do not need translating does potentially pose a threat to the translation profession. Desjardins (2017) argues that if non-text-based communication like the use of emojis continues to develop in importance then the need for translation could diminish and calls for translator training to look beyond the verbal. However, the use of images rather than text in global advertising campaigns to overcome the challenge of adapting marketing to different linguistic and cultural contexts is not at all a new strategy (e.g., see Adab \& Valdés, 2004; de Mooij, 2005).

\section{Conclusions and Implications}

This study analyzed a new area of translation practice in the soccer industry and is innovative in two ways. Firstly, it contributes to minimal existing research into the new area of social media and translation, especially from the perspective of professional practice. Secondly, the interrogation of translation and Twitter in the soccer industry begins to address 
the research gap in the field of sport and communication of communication challenges faced by migrant players and coaches and the clubs and associations that host them in the global sports industry.

In line with Hutchins' (2014) recommendation that research into Twitter should focus more centrally on commodification, the argument underlines the significance of the intersection between professional translation work on Twitter and the highly PR-conscious global industry/culture of soccer. It has demonstrated that when tweets produced by soccer players, their agents, soccer clubs, and governing organizations are translated, there is a constant eye kept on the management of "authentic" backstage (after Goffman, 1959) online identity and its economic potential. A common concern is optimizing visibility to maximize potential revenue. This sensitivity of clients to promoting and protecting the image of their brand manifests itself in tweet translations, both in the industry norm of fluent translation but also in the highly unusual practice of deliberate non-fluent translation. In the latter practice, the concern is not with clear communication but with the preservation of authentic identity in language expression, despite the common assumption about who actually authors tweets in celebrity contexts. Features that reinforce the marketable value of authenticity in social media are misspellings and grammatical errors which are detected both in tweet translation and proofreading practice. These practices produce the kind of foreignization in translation that Venuti advocates to render source culture and language visible because players' or coaches' "foreignness" remains evident through non-fluent language use, as implicitly does their culture, and the process of translation. The time pressure that the translators of tweets work under, especially in live in-match tweeting, can affect fluency and accuracy, and technical factors can affect visibility. On the one hand, the speed of technology creates audience expectations that assume automatic translation is the tool being used, making rapid human translators invisible. On the other hand, delays in producing and posting translated tweets 
caused by technology make the presence of translation more visible than is usually desirable in the industry.

The very particular practice of translating tweets in a non-fluent way or maintaining awkward host-language features of a migrant player or coach's tweets is one striking example in a range of ways in which players and clubs in particular have been shown to glocalize in the way they approach tweet translation, tailoring their brand to differentiated markets, "securing a profitable global presence [...] [by] operating in the languages of the local" (Giulianotti \& Robertson, 2007, p. 34). In fact, the range of practices identified reflect a spectrum of globalizing (homogenizing) and glocalizing strategies. On the one hand there is the globalizing non-translation of English language hashtags. On the other hand local linguistic identity is preserved both in the use of bilingual Twitter accounts where two languages appear side by side, and in the use of parallel monolingual accounts. In the latter local identity is preserved in one account and localized language audiences are targeted in other accounts, or indeed in the need for additional linguistic knowledge to decode emojis. There is, however, far more evidence in the data of glocalizing practices than of globalizing practices indicating that tweet translation reflects the general global commercial behavior of major sports teams as transnational corporations described by Giulianotti and Robertson.

The findings of the current investigation develop understanding of digital interactions in contexts of linguistic diversity in professional soccer. They encourage sports marketing departments to reflect on their use of Twitter and translation to better inform their brand management. The findings provide a platform for more research into the professional practice of social media translation in other linguistically diverse sectors of the sports industry, and indeed in other commercial contexts. For example, a more comprehensive study of how the non-linear hashtag is handled across linguistic and cultural barriers in sport communication contexts would help determine to what extent general practice reflects homogenizing 
globalization processes or more the successful glocalizing business practices that dominate in the data discussed above. The examples presented above of the use of emojis in soccer club tweets and their "translation" invite a broader assessment from the perspective of translation practitioners of the effect on the translation profession of the need for increased visual literacy in decoding communication on social media. Does this evolution in communication genuinely threaten the translation profession as Desjardins claims?

The role of translation in the management of the backstage identity of soccer players and coaches on social media uncovered here adds another layer to the processes which contribute to the construction of online identity, and the marketability of that identity. It would be valuable to extend the exploration to other linguistically diverse sectors of the sports industry. And indeed to wider professional contexts such as the entertainment industry where the management of the performance of identity on social media concerns celebrities who speak more than one language. Would the overall glocalizing approach found in professional soccer be replicated in the translation of social media in these two contexts?

In casting a light on Twitter translation in the context of professional soccer, this study has merely scratched the surface of the virtually uninvestigated communication challenges faced by migrant players and coaches in the soccer industry, and of the clubs, associations and fan bases that host and support them. Despite the extremely diverse character of most global professional sport communities (audiences, sponsors, owners, and athletes and coaches), such work is highly unusual in the context of sport and communication studies. The complex navigation between local and global identity detailed above in Twitter translation is likely to also feature prominently in a range of communication contexts for these migrants, between first language and host language, and potentially via shared languages that bridge the gap between the two. Whether it be communication in professional situations such as recruitment medicals, media work, or training, or in extra-professional 
situations such as finding a home, understanding tax laws, or using health services, there is much more to be discovered to understand and support communication for these migrants. 


\section{References}

ACAS. (2018, January). Social media in the workplace. Advisory, Conciliation and Arbitration Service. Retrieved from: http://www.acas.org.uk/index.aspx?articleid=3375

Adab, B., \& Valdés, C. (2004). Key debates in the translation of advertising material. The Translator, 10(2), 161-177. https://doi.org/10.1080/13556509.2004.10799175

Alshenqeeti, H. (2016). Are emojis creating a new or old visual language for new generations? A socio-semiotic study. Advances in Language and Literary Studies, 7(6), 56-69. http://dx.doi.org/10.7575/aiac.alls.v.7n.6p.56

Associated Press. (2015, December 16). 2014 World cup final attracted 1.01 billion viewers, FIFA says. Retrieved from http://www.espn.co.uk/football/world-cup$\underline{\text { soccer/story/2759180/fifa-reports-101-billion-viewers-for-2014-world-cup-final }}$

Baines, R. (2018). Translation and interpreting for the media in the English Premier League: Elite level football, power, and translation. In S. Baumgarten \& J. CornellàDetrell (Eds.), Translation and Global Spaces of Power (pp. 179-193). Bristol: Multilingual Matters.

Bassnett, S. (2005). Bringing the news back home: Strategies of acculturation and foreignisation. Language and Intercultural Communication, 5(2), 120-130. http://dx.doi.org/10.1080/14708470508668888

Bennett, S. (2011). 39\% of all tweets are in English, but Arabic now fastest-growing language on Twitter. Adweek. Retrieved from https://www.adweek.com/digital/twitter-language-share/

Billings, A., \& Hardin, M. (Eds.). (2014). Routledge handbook of sport and new media. London/New York: Routledge.

Carter, T. (2011). In foreign fields: The politics and experiences of transnational sport 
migration. London: Pluto Press. http://dx.doi.org/10.1111/jlca.12057

Danesi, M. (2017). The semiotics of emoji: The rise of visual language in the age of the Internet. London/New York: Bloomsbury.

Desjardins, R. (2013). Social media. In Y. Gambier \& L. van Doorslaer (Eds.), Handbook of translation studies (Vol.4, pp. 156-159). Amsterdam: John Benjamins.

Desjardins, R. (2017). Translation and social media. Basingstoke: Palgrave Macmillan.

Drugan, J. (2013). Quality in professional translation: Assessment and improvement. London/New York: Bloomsbury.

Eriksen, T. H. (2014). Globalization: The key concepts. London/New York: Bloomsbury.

Evans, V. (2017, July 6). Why we need emojis. Nautilus, 50. Retrieved from http://nautil.us/issue/50/emergence/why-you-need-emoji

Fuchs, C. (2017). Social media: A critical introduction. London: Sage.

Georgakopoulou, A. \& Spilioti, T. (Eds.). (2015). Routledge handbook of language and digital communication. London/New York: Routledge.

Gilchrist, P. (2005). Local heroes or global stars. In L. Allison (Ed.), The global politics of sport: The role of global institutions in sport (pp. 107-126). London: Routledge.

Giulianotti, R., \& Robertson, R. (2007). Sport and globalization: Transnational dimensions. Global Networks, 7(2), 109-112. http://dx.doi.org/10.1111/j.1471-0374.2007.00159.x

Goffman, E. (1959). The presentation of self in everyday life. New York: Doubleday. Hale, S., \& Napier, J. (2013). Research methods in interpreting. London/New York: Bloomsbury.

Hambrick, M., Simmons, J., Greenhalgh, G., \& Greenwell, C. (2010). Understanding professional athletes' use of Twitter: A content analysis of athlete tweets. International Journal of Sport Communication, 3(4), 454-471. http://dx.doi.org/ 10.1123/ijsc.3.4.454 
Hutchins, B. (2010). The acceleration of media sport culture. Information, Communication \& Society, 14(2), 237-257. http://dx.doi.org/10.1080/1369118X.2010.508534

Hutchins, B. (2014). Twitter: Follow the money and look beyond sports. Communication \& Sport, 2(2), 122-126. http://dx.doi.org/10.1177/2167479514527430

Johnson, S. (2016, January 29). Revealed: QPR's 27-page rulebook on how players should behave... and what happens if they don't. Evening Standard. Retrieved from https://www.standard.co.uk/sport/football/revealed-qprs-27page-code-of-conductmanual-given-to-every-new-signing-on-arrival-a3168076.html

Jones, C., \& Steckelmacher, S. (2012, October 24). It's a funny old game: you say tomato, I say ‘solanum Lycopersicum’. Marca. Retrieved from http://www.marca.com/blogs/itsafunnyoldgame/2012/10/24/you-say-tomato-i-saysolanum.html

Kaufman, A. (2013, November 23). For Hollywood's social media managers, tweeting is a living. Washington Post. Retrieved from https://www.washingtonpost.com/entertainment/celebrities/for-hollywoods-socialmedia-managers-tweeting-is-a-living/2013/11/21/880110ce-5143-11e3-9fe0fd2ca728e67c_story.html?utm_term=.1d1d0bf9a84f)

Kelly, N. (2009, June 19). Freelance translators clash with LinkedIn over crowdsourced translation. Common Sense Advisory. Retrieved from http://www.commonsenseadvisory.com/Default.aspx ?Contenttype=ArticleDetAD\&ta bID $=63 \&$ Aid $=591 \&$ moduleId $=391$

Korzynski, P., \& Paniagua, J. (2016). Score a tweet and post a goal: Social media recipes for sports stars. Business Horizons, 59(2), 185-192. http://dx.doi.org/10.1016/j.bushor.2015.11.002 Kytölä, S., \& Westinen, E. (2015). “I be da reel gansta”: A Finnish footballer's Twitter 
writing and metapragmatic evaluations of authenticity. Discourse, Context and Media, 8, 6-19. http://dx.doi.org/10.1016/j.dcm.2015.05.001

Leary, M. R. (1995). Self-presentation: Impression management and interpersonal behavior. Madison, WI: Brown \& Benchmark.

Lebel, K., \& Danylchuk, K. (2012). How tweet it is: A gendered analysis of professional tennis players' self-presentation on Twitter. International Journal of Sport Communication, 5(4), 461-480. http://dx.doi.org/10.1123/ijsc.5.4.461

Maguire, J., \& Falcous, M. (2010). Sport and migration: Borders, boundaries and crossings. London: Routledge. doi: 10.1080/14927713.2011.555659

Mangiron, C., \& O’Hagan, M. (2006). Game localisation: Unleashing imagination with restricted translation. Journal of Specialised Translation. Retrieved from http://www.jostrans.org/issue06/art_ohagan_utf_test.php

Marwick, A., \& boyd, d. (2011). To see and be seen: Celebrity practice on Twitter. Convergence, 17(2), 139-158. http://dx.doi.org/10.1177/1354856510394539

Mocanu, D., Baronchelli, A., Perra, N., Gonçalves, B., Zhang, Q., \& Vespignani, A. (2013). The Twitter of Babel: Mapping world languages through microblogging platforms. PLoS ONE, 8(4). Retrieved from https://journals.plos.org/plosone/article?id=10.1371/journal.pone.0061981

de Mooij, M. (2005). Global marketing and advertising: Understanding cultural paradoxes. New York: Sage.

Parganas, P., Anagnostopoulos, C., \& Chadwick, S. (2015). 'You'll never tweet alone': Managing sports brands through social media. Journal of Brand Management, 22(7), 551-568. http://dx.doi.org/10.1057/bm.2015.32

Pegoraro, A., \& Jinnah, N. (2012). Tweet 'em and reap 'em: The impact of professional 
athletes' use of Twitter on current and potential sponsorship opportunities. Journal of Brand Strategy, 1(1), 85-97.

Poli, R. (2014). International migration of professional footballers. In J. Goddard and P. Sloane (Eds.), Handbook on the economics of football (pp. 227-237). London: Edward Elgar.

Price, J., Farrington, N., \& Hall, L. (2013). Changing the game? The impact of Twitter on relationships between football clubs, supporters and the sports media. Soccer \& Society, 14(4), 446-461. http://dx.doi.org/10.1080/14660970.2013.810431

Robertson, R. (2012). Globalisation or glocalisation? Journal of International Communication, 18(2), 191-208. http://dx.doi.org/10.1080/13216597.2012.709925

Roderick, M. (2006). A very precarious profession: Uncertainty in the working lives of professional footballers. Work, Employment and Society, 20(2), 245-265. http://dx.doi.org/10.1177/0950017006064113

Ruthven, G. (2016, October 25). \#AgainstModernFootball - footballers on Twitter. Yahoo Sport. Retrieved from https://uk.sports.yahoo.com/news/againstmodernfootballfootballers-on-twitter-103029552.html

Sanderson, J. (2013). Stepping into the (social media) game: Building athlete identity via Twitter. In R. Luppiccini (Ed.), Handbook of research on technoself: Identity in a technological society (Vol. 2, pp. 711-730). Hershey, PA: IGI Global.

Schäler, R. (2010). Localization and translation. In Y. Gambier \& L. Van Doorslaer (Eds.), Handbook of translation studies (Vol. 2, pp. 209-214). Amsterdam: John Benjamins.

Thomas, S. (2014). Celebrity in the 'Twitterverse': History, authenticity and the multiplicity of stardom. Situating the 'newness' of Twitter. Celebrity Studies, 5(3), 242-255. http://dx.doi.org/10.1080/19392397.2013.845962

Vaast, E., \& Kaganer, E. (2013). Social media affordances and governance in the 
workplace: an examination of organizational policies. Journal of Computer-Mediated Communication, 19(1), 78-101. http://dx.doi.org/10.1111/jcc4.12032

Venuti, L. (2008). The translator's invisibility: A history of translation. London/New York: Routledge.

Venuti, L. (2010). Translation as cultural politics: Régimes of domestication in English. In M. Baker (Ed.), Critical readings in translation studies (pp. 65-79). London/New York: Routledge. 


\section{Gabriel Jesus}

@gabrieljesus33

Follow

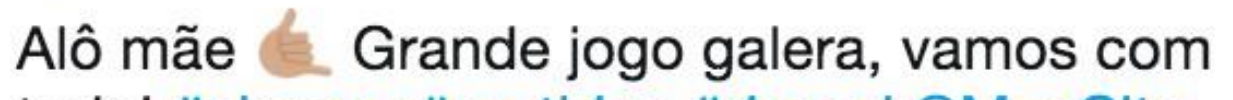
tudo! \#alomae \#gratidao \#doperi @ManCity

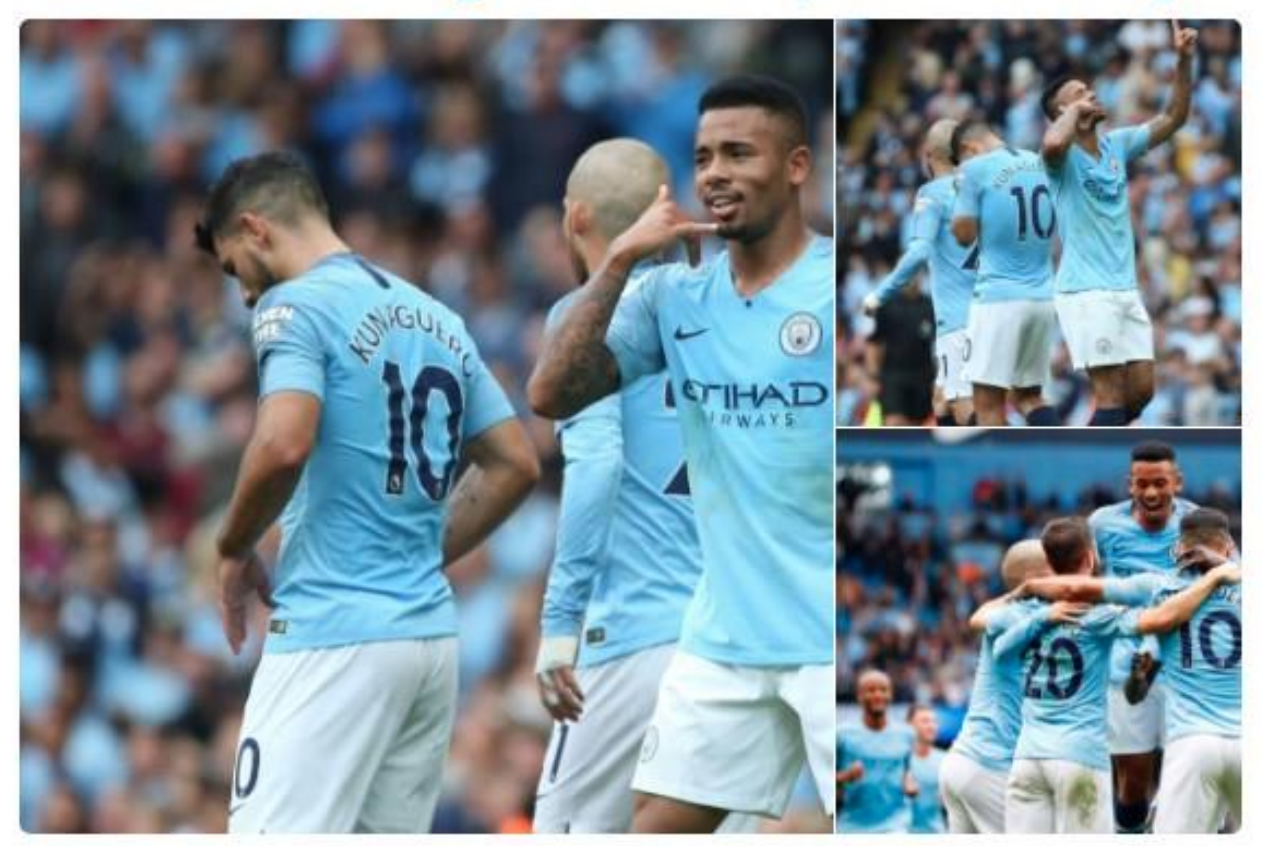

5:26 PM - 19 Aug 2018

Figure 1 (Literal translation: "Hello Mother. Great game guys, let's go with everything!"). $210 \times 202 \mathrm{~mm}(72 \times 72$ DPI $)$ 


\section{Today match against NY red bulls and after} come back to london!!! Nice weekend everyone

7:08 PM - 26 Jul 2014

Figure 2

$212 \times 68 \mathrm{~mm}(72 \times 72 \mathrm{DPI})$ 
Another important one tonight . Matchday

Mais um jogo está noite do campeonato inglês. Deus... instagram.com/p/Bcm83MfgHEO/

6 Translate from Portuguese

4:18 PM - 12 Dec 2017

Figure 3 
ACF Fiorentina Bacffiorentina - May 20

Pareggio immediato dei rossoneri con \#Calhanoglu su punizione al 23'

\#MilanFiorentina 1-1

6 Translate Tweet

Q 1 ᄂ

ACF Fiorentina (Bacffiorentina - May 20

23: Goal for AC Milan. Calhanoglu levels it up immediately with a free-kick. It's 11 at San Siro \#MilanFiorentina

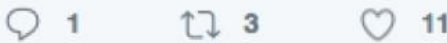

Figure 4 


\section{(ix) Olympique de Marseille} @OM_Officiel

\section{Prolongation de contrat}

Arrivé à l'OM en 2015, Yohann Pelé a prolongé, son contrat avec l'OM jusqu'en 2020

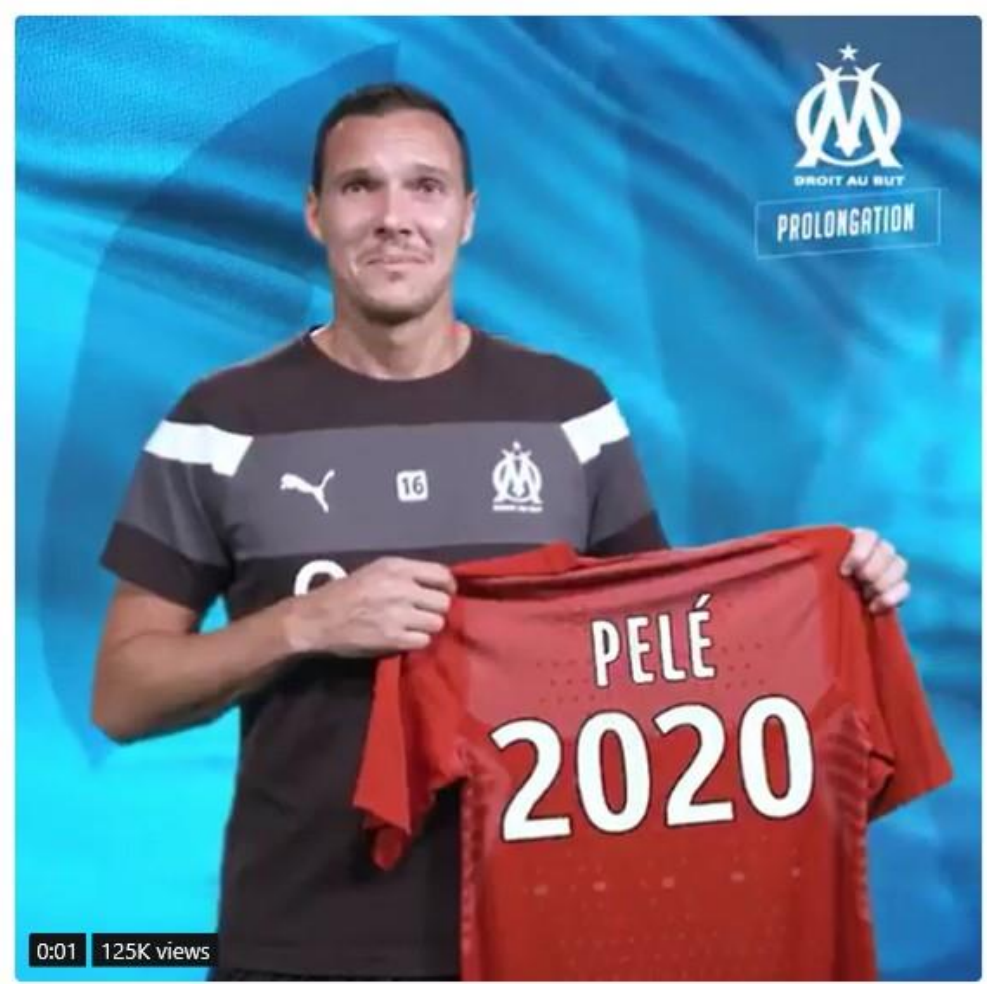

1:01 AM - 21 Jul 2018

834 Retweets 3,887 Likes $\quad 808080$

(Literal translation: "Arrived at OM in 2015, Yohann Pelé has extended his contract with OM until 2020"). 
Come on in \& stay a while, Yohann Pelé! Congratulations on your contract extension through 2020

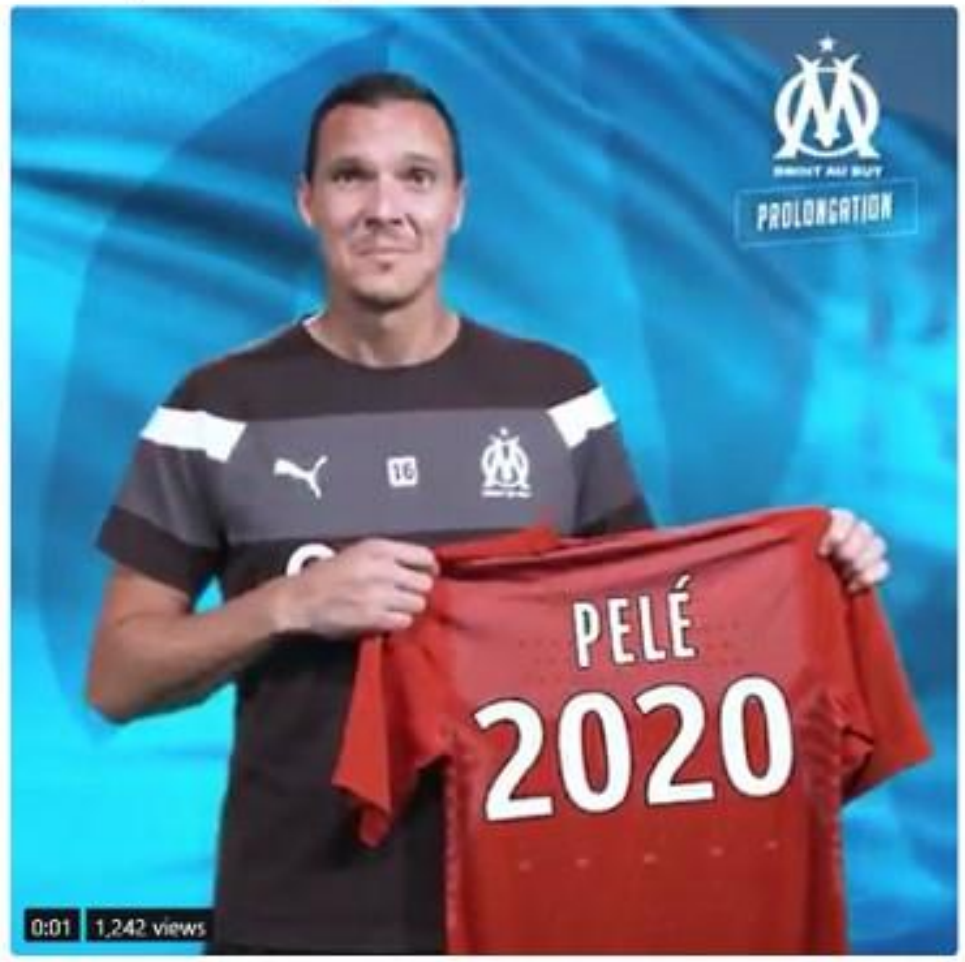

11:45 AM - 21 Jul 2018

Figure 5 


\section{Manuel Lanzini}

@manulanzini

3 years ago, I only want to say thanks to fans a 2 \#COYI

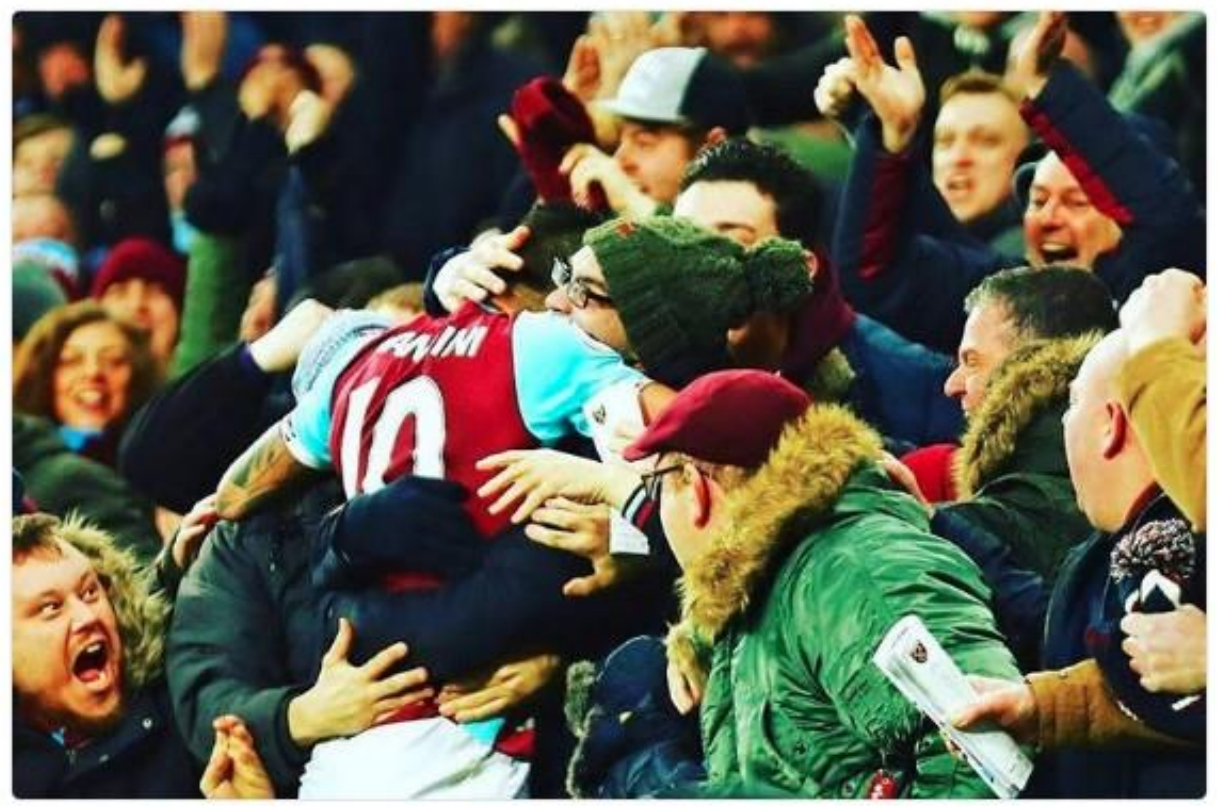

8:32 PM - 22 Jul 2018

Figure 6

$215 \times 201 \mathrm{~mm}(72 \times 72$ DPI $)$ 


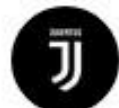
JuventusFC @juventusfc

In Italia siete pronti per andare a dormire? Qui in $\underline{\underline{\underline{\underline{E}}}}$ ci stiamo appena scaldando... (5) \#CONTAJUS

6 Translate Tweet

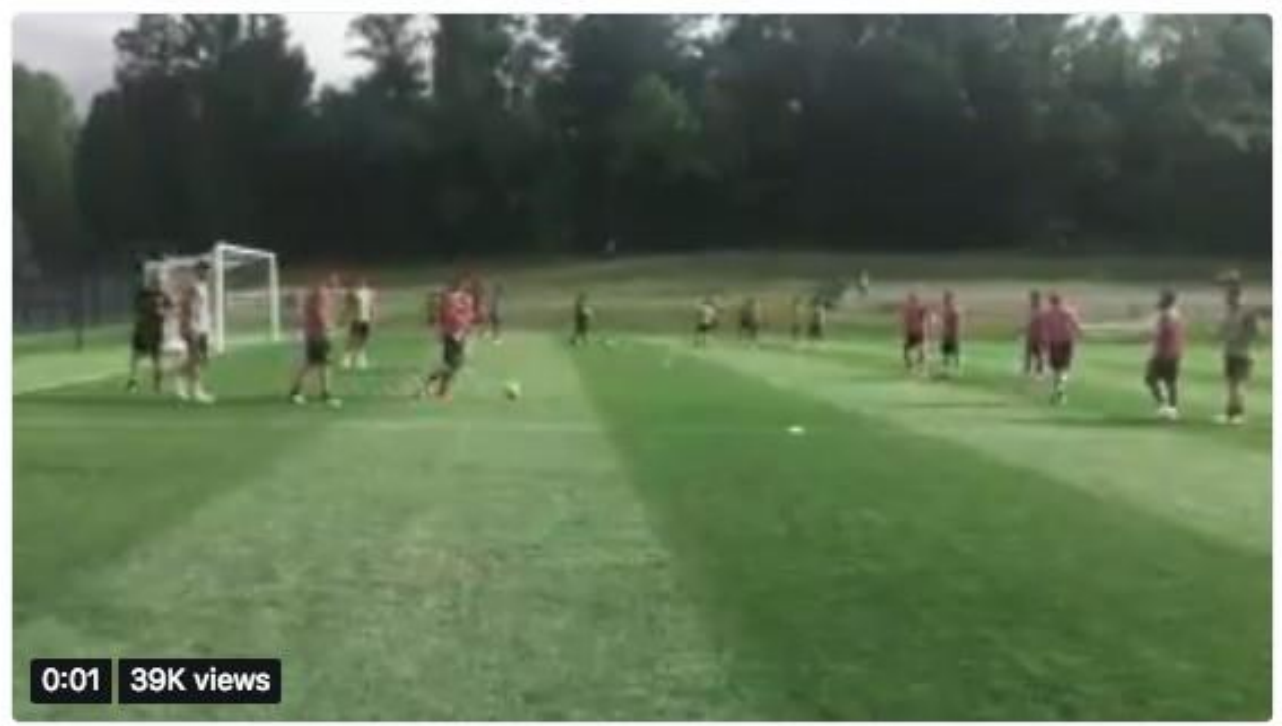

10:53 PM - 24 Jul 2018

Figure 7 (Literal translation: "Are you ready to go to sleep in Italy? Here in America, we are just warming up..."). 


\section{Bayer 04 Leverkusen}

@bayer04_en

\section{Kai Havertz now has two assists in the game today!}

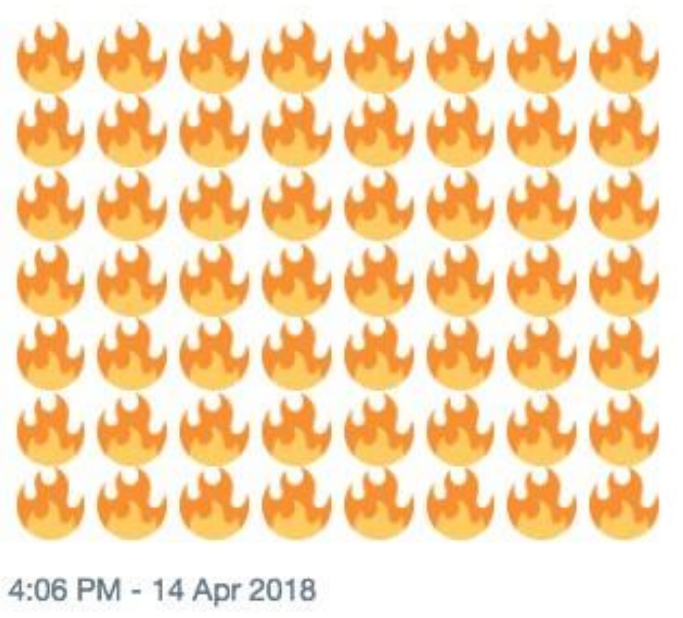

Figure 8

$212 \times 159 m m(72 \times 72$ DPI $)$ 